\title{
Optimization and numerical simulation analysis for molded thin-walled parts fabricated using wood-filled polypropylene composites via plastic injection molding
}

\begin{abstract}
Plastic injection molding is discontinuous and a complicated process involving the interaction of several variables for control the quality of the molded parts. The goal of this research was to investigate the optimal parameter selection, the significant parameters, and the effect of the injection-molding parameters during the post-filling stage (packing pressure, packing time, mold temperature, and cooling time) with respect to in-cavity residual stresses, volumetric shrinkage and warpage properties. The PP $\mathbb{A}$ (6010 wt $\%$ wood material is not suitable for molded thin-walled parts. In contrast, the PPIAII $\mathrm{wt} \%$ material was found to be the preferred type of lignocellulosic polymer composite for molded thin-walled parts. The results showed the lower residual stresses approximately at $20.10 \mathrm{MPa}$ and have minimum overpacking in the ranges of $\bar{i} 0.709 \%$ to $\bar{i} 0.174 \%$ with the volumetric shrinkage spread better over the part surface. The research found that the packing pressure and mold temperature are important parameters for the reduction of residual stresses and volumetric shrinkage, while for the reduction of warpage, the important processing parameters are the packing pressure, packing time, and cooling time for molded thin-walled parts that are fabricated using lignocellulosic polymer composites.
\end{abstract}

Keyword: Plastic injection molding; Packing pressure; Mold temperature; Thin-walled parts; Injection moulding 